\title{
Intrinsic dissipation in high-frequency micromechanical resonators
}

\author{
P. Mohanty, * D. A. Harrington, K. L. Ekinci, Y. T. Yang, M. J. Murphy, and M. L. Roukes \\ Condensed Matter Physics 114-36, California Institute of Technology, Pasadena, California 91125
}

(Received 2 January 2002; Revised 26 March 2002; published 20 August 2002)

\begin{abstract}
We report measurements of intrinsic dissipation in micron-sized suspended resonators machined from single crystals of galium arsenide and silicon. In these experiments on high-frequency micromechanical resonators, designed to understand intrinsic mechanisms of dissipation, we explore dependence of dissipation on temperature, magnetic field, frequency, and size. In contrast to most of the previous measurements of acoustic attenuation in crystalline and amorphous structures in this frequency range, ours is a resonant measurement; dissipation is measured at the natural frequencies of structural resonance, or modes of the structure associated with flexural and torsional motion. In all our samples we find a weakly temperature dependent dissipation at low temperatures. We compare and contrast our data to various probable mechanisms, including thermoelasticity, clamping, anharmonic mode-coupling, surface anisotropy and defect motion, both in bulk and on surface. The observed parametric dependencies indicate that the internal defect motion is the dominant mechanism of intrinsic dissipation in our samples.
\end{abstract}

DOI: 10.1103/PhysRevB.66.085416

PACS number(s): 62.65.+k, 62.20.Dc, 62.40.+i

\section{INTRODUCTION}

Dissipation in a mechanical resonator describes the relaxation or loss of energy contained in a resonant mode to the external environment coupled to the structure as well as to the other resonant modes. ${ }^{1}$ In spite of the obvious importance of single-crystal resonators in areas such as gravity-wave detection and high-precision measurements, ${ }^{2-10}$ their acoustic properties are not very well understood. The cause of dominant dissipation, for example, in resonators of any size at low temperatures, is not known, the primary reason being the lack of extensive experiments on acoustic energy loss in single-crystal resonators at low temperatures. In particular, whether the observed dissipation is intrinsic to the material of the resonator or due to the extrinsic experimental artifacts is still under debate. Furthermore, the reduction of the size of the resonators to the micron scale is believed to result in dissipation dominated by finite-size effects due to the large surface contributions. The nature of these surface contributions, either intrinsic or artifactual, has not yet been studied. Recent work has begun to address this issue, ${ }^{11-25}$ but a clear picture still remains elusive.

At the heart of understanding what determines dissipation in micron-sized systems is the identification of the dominant dissipation mechanism and its scaling with system size. There exists a host of identifiable mechanisms, both intrinsic and extrinsic, which play an important role in the dissipation of a micromechanical structure. Some of the examples are the loss due to clamping, thermoelastic process, gas friction, and the coupling to various dislocations and localized internal defects. Typically, dissipation is determined by a combination of many different mechanisms which dominate in different parts of the parameter space. Since dissipation is the rate at which energy is lost from a resonant mode to various environments coupled to the mode, the dominant mechanism is the one with the highest rate of energy loss. The problem, however, is in identifying the mechanisms as well as discerning the intrinsic from the extrinsic, which poses an experimental challenge. Since different mechanisms have different dependencies on various experimental parameters such as temperature, pressure, and magnetic field, it is possible to determine the dominant mechanism by ruling out the other mechanisms through their parametric dependence or lack thereof. Experimentally, this is crucial to the identification of an unknown mechanism. In a certain region of parameter space, all the dependencies must be accounted for by this mechanism alone, rather than catering different mechanisms to the dependencies on different parameters.

Pertaining to the study of dissipation in single crystals, only a handful of experiments have been performed. Kleiman and co-workers ${ }^{26}$ have measured sound velocity and attenuation in macroscopic single-crystal silicon resonators in the millikelvin temperature range. The size of their resonators in the centimeter range corresponds to resonant modes in low kilohertz range. Quality factor of $\sim 10^{6}$ for the torsional modes in their samples is suggestive of a mechanism of dissipation other than the clamping loss. The temperature dependence in their samples is similar to that in vitreos silica, and hence they concluded that the observed dissipation is due to two-level systems, the dominant mechanism in vitreos silica. ${ }^{27,28}$ Mihailovich and co-workers ${ }^{17}$ have studied the low temperature dissipation and shift in the sound velocity in single-crystal resonators of similar sizes. Furthermore, by introducing boron impurities into the resonator they found a further increase of dissipation rate with increasing doping level of boron. They concluded that the intrinsic dissipation of undoped structures is governed by nonglassy (electronic) defects as well. In a series of elegant experiments, Greywall and co-workers ${ }^{21}$ have studied and potentially identified an anomalous contribution to the low temperature dissipation, in the form of distinct peaks, in single-crystal silicon resonators. They have also confirmed that the low temperature dissipation is, otherwise, featureless and almost independent of temperature. Pohl and co-workers ${ }^{29}$ have also studied various acoustic properties in large single crystal structures made from silicon. In a series of experiments from the groups of Craighead and Parpia ${ }^{13,14,18,22,23}$ intrinsic dissipation studied in nano-electro-mechanical resonators (NEMS) in the 1-10 $\mathrm{MHz}$ range has been identified with "surface and nearsurface phenomena." Temperature dependence of dissipation 
in these silicon devices revealed a peak in the range of 160 $190 \mathrm{~K}$. These experiments also revealed that the metallic electrodes on the surface of these devices used for measurement did not contribute to the temperature dependence. Furthermore, dissipation in two single-paddle oscillators with frequencies $2.3 \mathrm{MHz}$ and $6.6 \mathrm{MHz}$ displayed a very weak temperature dependence or saturation below $30 \mathrm{~K}^{22}$ In a series of recent experiments by Yang, Ono, and Esashi, ${ }^{24}$ the surface contributions to dissipation has been studied in ultrathin single-crystal silicon cantilevers. They show that dissipation linearly increases with cantilever thickness.

In the above-mentioned experiments, both electronic and nonelectronic defects have been identified, with some success, as the main contributors to the intrinsic dissipation, and the relevance of enhanced surface-to-volume ratio has been emphasized. The electronic nature of the defects was identified through a magnetic-field dependence present in the works of both Mihailovich and co-workers ${ }^{17}$ and Greywall and co-workers. ${ }^{21}$ However, various other extrinsic mechanisms must be taken into experimental consideration in a complete study of the identification of the dissipation mechanism. In addition, the small size of the resonators is expected to introduce nontrivial effects such as the finite-size effect in dissipation, since the surface contributions become nonnegligible compared to the volume contributions. A complete study of acoustic properties of such devices at low temperatures has not yet been done.

With regard to the dominating mechanism of dissipation in micron- and submicron-sized structures, a host of questions have emerged from these recent experiments in literature. Some of these questions are enumerated below:

(1) What determines the intrinsic dissipation in micron and submicron structures?

(2) Why does the quality factor $Q$ (inverse of dissipation) usually scale linearly with size in small structures $^{14,17,19,21,24,26,29-35}$ as shown in Fig. 1?

(3) Why does the quality factor of the micron-sized resonators always lie in the range of $10^{3}-10^{5}$ at liquid helium temperatures?

(4) Is it possible to increase the quality factor of these resonators substantially, by orders of magnitude?

In this paper, we report extensive measurements of dissipation in micron-sized single crystal GaAs and Si resonators. We have measured the temperature and magnetic field dependence of the internal dissipation $\left(Q^{-1}\right)$ and the shift in resonance frequency $\delta f / f$ in the range of normal mode frequencies between 0.5 and $2.8 \mathrm{MHz}$ in GaAs resonators, and up to $13 \mathrm{MHz}$ in Si resonators. These data exhibit signatures of internal defect motion in the temperature dependence of $Q^{-1}$ and $\delta f / f$ in the high temperature part, in the magnetic field dependence of $Q^{-1}$, and in the hysteretic behavior of both $Q^{-1}$ and $\delta f / f$ on thermal cycling. The significant contribution to dissipation from the internal defects is further confirmed by the observed size dependence of $Q^{-1}$ in that the dissipation, or $Q^{-1}$, in systems smaller than a critical size shows a well defined inverse linear dependence with size. This linear dependence is expected due to the increasing relevance of the defects at the surface for smaller resonators.

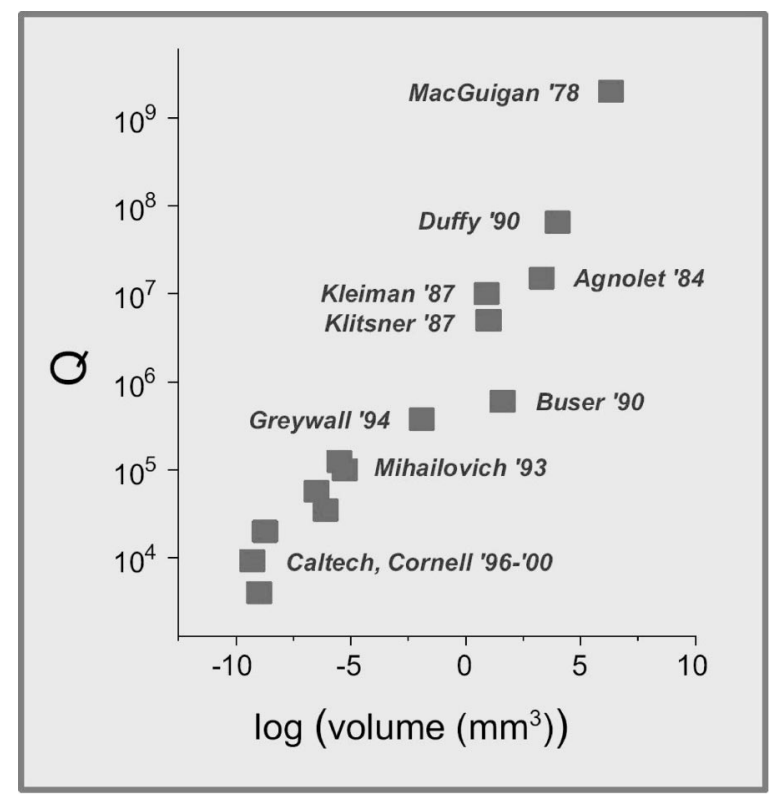

FIG. 1. The overall trend of the size dependence of quality factor in mechanical resonators.

In addition, we find the behavior of both $Q^{-1}$ and $\delta f / f$ in the temperature range of $4-40 \mathrm{~K}$ to be similar to the discrepancies in acoustic and dielectric properties of kilohertz frequency resonators measured below $100 \mathrm{mK}$. In this paper, we discuss in detail the high temperature data for a prototype GaAs sample, representative of six other similar samples studied in detail. Single crystal silicon structures studied in this range of frequency also displayed similar trends.

In this paper we establish the following phenomenological features relevant to the identification of the intrinsic dissipation mechanism in our micron-sized GaAs and $\mathrm{Si}$ resonators. These features may be universal to all GaAs and $\mathrm{Si}$ resonators of comparable sizes.

(i) Dissipation $\left(Q^{-1}\right)$ and the shift $(\delta f / f)$ in the resonance frequency is weakly temperature dependent in the low temperature range (below $40 \mathrm{~K}$ ).

(ii) Dissipation quadratically increases with increasing magnetic field: $Q^{-1}(B)=Q^{-1}(B=0)+a B^{2}$.

(iii) Both the dissipation and the resonance frequency shift are hysteretic on thermal cycling. More often than not, the dissipation decreases slightly (less than a few percent) on annealing between $4 \mathrm{~K}$ and $300 \mathrm{~K}$.

(iv) Dissipation displays aging on thermal cycling. With increasing thermal cycles, it approaches a constant value.

(v) The dissipation in all the structures reported in this paper is independent of the driving amplitude. In other words, the measurements are done in the linear regime.

In our experiments we identify internal dynamic defects as the dominant dissipation mechanism in our samples. Additionally, we rule out other extrinsic mechanisms. In Sec. II, we briefly describe the experimental technique followed by a discussion of the data. Various checks performed in our experiments as well as the comparison of the data to different 


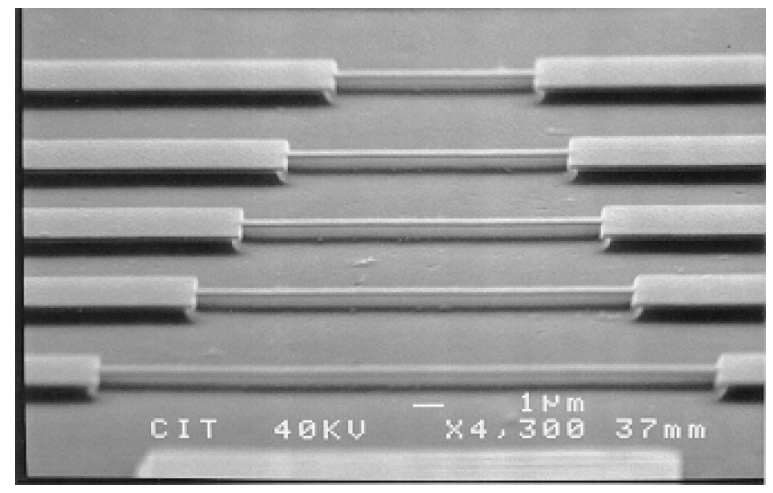

FIG. 2. A series of silicon beams with transverse dimensions in the submicron scale.

extrinsic mechanisms are summarized in Sec. IV. In Sec. V, we discuss the relevance of internal defects and $D-x$ centers to the dissipation in GaAs. Finally, we discuss various mechanisms important to the understanding of our data. The possible role of configurational entropy arising from the large degree of degeneracy in the configuration of the internal defects is argued to be relevant to the observation of hysteresis, aging, and the weak temperature dependence.

\section{EXPERIMENTAL TECHNIQUE}

The micrograph of a series of silicon beams is shown in Fig. 2, and the measured quality factor in such a series of silicon beams with varying lengths is shown in Fig. 3. In order to discern the intrinsic mechanism of dissipation, the extrinsic mechanisms have to be minimized or avoided. As we will discuss later in the paper, the appropriate structure for a comprehensive study is a two-element torsional resonator. The scanning electron micrograph of a prototypical twoelement torsional GaAs resonator is displayed in Fig. 4. The GaAs resonators are fabricated from single-crystal GaAsAlGaAs-GaAs heterostructures. Four normal modes of motion are observed at frequencies $0.62,1.02,1.28$, and 2.75 $\mathrm{MHz}$. They correspond to, in order, the fundamental flexure:

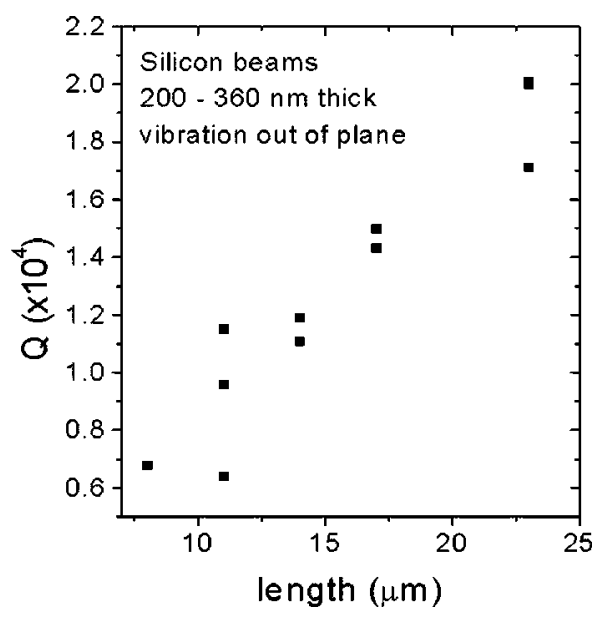

FIG. 3. Size dependence of quality factor measured in a series of silicon beams. The data displays a linear dependence of $Q$ on size.

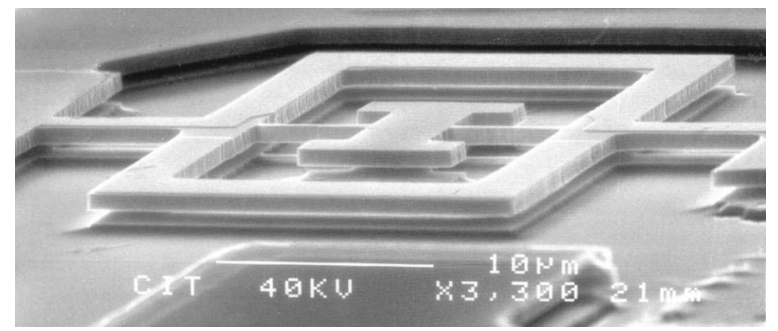

FIG. 4. SEM image of the suspended GaAs resonator. The submicron-sized thin rod connecting the outer and inner torsion elements supports the strain for the antisymmetric torsion, mode C.

mode A, symmetric torsion: mode B, antisymmetric torsion: mode $\mathrm{C}$ and the second flexure: mode $\mathrm{D}$. Antisymmetric torsion in mode $\mathrm{C}$ corresponds to a strain localized mostly in the metal-free inner torsion rods whose size varies between 0.2 and $1.0 \mu \mathrm{m}$ for the six samples studied in detail. The resonators have a lateral extent of $\sim 25 \mu \mathrm{m}$ and a thickness of $800 \mathrm{~nm}$. A gold electrode is deposited on the outer torsional element for the magnetomotive excitation and detection of the motion. ${ }^{20}$ The measurements were done inside the bore of an $8 \mathrm{~T}$ superconducting magnet. The samples were placed inside the vacuum can of the cryostat; the vacuum can was evacuated with a turbomolecular pump. The chamber pressures were measured at $<1 \mathrm{mTorr}$ at room temperature. The cryopumping of the walls in liquid helium further reduced the pressure inside the can by at least a factor of 100 . The temperature sweeps were done by heating the sample resistively.

\section{A. Measurement by the magnetomotive technique}

There is a host of techniques to measure the motion of micron-sized or nanoscale mechanical resonators. The optical technique and capacitive technique are the two most common methods, which involve actuation and detection of motion by an optical (laser) signal and a capacitivelycoupled electric field, respectively. However, as the structure gets smaller and more complicated, it becomes increasingly difficult to use these techniques for force or displacement detection. The inherent problem with optical detection is that a nanomechanical sample structure, or a specific part of it, cannot be individually targeted because the minimum spot size is usually much larger than the typical device size. Second, noise induced by the optical (laser) beam, for example, shot noise and nonlinear heating, is substantially large if accurate measurement of motion is desired. The capacitive method, on the other hand, suffers from the following main problems: (a) it restricts the design of the device since an optimum capacitance between a detector/actuator plate and the surface of the sample is desired for an optimum signal size, (b) parasitic capacitance is difficult to account for, and (c) at high frequencies, a large capacitive coupling to the actuator or detector provides a short circuit for the highfrequency current, reducing the control and access to the device itself.

We have measured our mechanical devices by the magnetomotive technique. In this technique, the resonator, or a spe- 
cific part of the resonator, is excited by the application of a drive current $I_{D}$ through an electrode on its surface in presence of a magnetic field $B$ perpendicular to the length $L$ of the current-carrying electrode. Motion is detected by measuring the induced emf in the electrode. Let us consider a simple doubly-clamped beam structure, shown in Fig. 2. (The following analysis describes, without any loss of generality, flexural as well as torsional motion of complicated structures like the one shown in Fig. 4.) The motion of the doubly-clamped beam is characterized by the displacement variable $y(x, t)$ at a certain point $x$ along the beam. Its motion is described by a damped harmonic oscillator with an effective mass $m$ and effective spring constant $k$,

$$
m \frac{d^{2} y(t)}{d t^{2}}+\gamma m \frac{d y(t)}{d t}+k y(t)=F(t) ;
$$

the damping coefficient $\gamma$ represents the loss of energy due to the coupling to internal and external degrees of freedom. $F(t)$ is the time-dependent driving force. For a complicated structure or a structure with many modes, the mass term $m$ depends on the mode shape. The effective spring constant $k$ depends on the displacement point $y(t)$ where motion is measured-implying, for a specific point $y(t)$ along the beam, one obtains a specific spring constant $k$, assuming uniform force applied to the structure. Because of the displacement of the structure $y(x, t)$ in the lateral direction, there will be an induced emf in the electrode,

$$
V_{\mathrm{emf}}(x, t)=\frac{d y(x, t)}{d t} B \sin \theta(x),
$$

where $\theta$ is the angle between the field and the electrode. An average emf can be obtained by integrating the response along the length of the electrode,

$$
V_{\mathrm{avg}}(t)=\xi L B \frac{d y(t)}{d t} .
$$

The constant $\xi$ can be explicitly calculated for a given structure: for the fundamental flexural mode of a doubly-clamped beam, $\xi \simeq 0.53$ at the center of the beam. The frequency dependence of the induced emf is important, as it can be directly measured by a vector network analyzer. The Fourier transform of $V_{\text {avg }}(t)$ is given by

$$
V_{\mathrm{avg}}(\omega)=\frac{i \omega \omega_{0}^{2} \xi L L_{\mathrm{beam}} B^{2} / k}{\omega_{0}^{2}-\omega^{2}+i \gamma \omega} I_{d}(\omega) .
$$

The Lorentzian line shape is a generic form for a damped harmonic oscillator, centered at the (natural) resonance frequency of the mode, $\omega_{0}=\sqrt{k / m}$. The maximum response is at this central frequency, and it is inversely proportional to the damping coefficient $\gamma$. The quality factor $Q$ is defined as $Q=\omega_{0} / \gamma$. Near the resonance, $\omega \simeq \omega_{0}$, the induced emf is given by

$$
V_{\mathrm{avg}}(\omega)=\frac{\xi L L_{\mathrm{beam}} B^{2} \omega_{0}}{k} Q I_{d}
$$

This expression connects the average motion-induced voltage $V_{\text {avg }}$ in the electrode to an applied current $I_{d}$, which prompts the following definition of a mechanical resistance:

$$
R_{m}=\frac{\xi L L_{\mathrm{beam}} B^{2} \omega_{0}}{k} Q .
$$

$R_{m}$ is in units of Ohm. In fact, the damped harmonic oscillator equation can be written as an equation for a damped LCR circuit. Such an analysis ${ }^{36}$ gives the following equivalent electrical-circuit elements for the mechanical motion,

$$
\begin{gathered}
R_{m}=\frac{\xi L L_{\text {beam }} B^{2} \omega_{0}}{k} Q, \\
C_{m}=\frac{k}{\xi L L_{\text {beam }} B^{2} \omega_{0}^{2}}, \\
L_{m}=\frac{\xi L L_{\text {beam }} B^{2}}{k} .
\end{gathered}
$$

This analysis is quite general in the sense that mechanical motion can be described in terms of equivalent electrical circuit parameters, and the physical meaning of the parameters also extends to the description of the mechanical system. The resistance $R_{m}$ is the dissipative part, naturally connected to the quality factor $Q$. Likewise, the capacitance $C_{m}$ can be important in describing the short-circuiting of the high-frequency field in relation to the true parasitic capacitance of the system.

\section{B. Noise and sensitivity considerations}

Noise induced by the measurement technique limits the sensitivity with which the mechanical properties of a resonator can be measured. Two major categories of noise are ambient noise (due to effects of temperature, pressure, etc.) and measurement noise (due to the measurement current or power, instrumental limitations, etc.). In addition, nonlinearity and other nonideal measurement schemes result in an inaccurate measurement. We have taken extensive precautions both in the design of the experiment and in the measurement to minimize the undesirable effects, some of which are discussed in section on extrinsic mechanisms. For the sake of completeness, we give a brief analysis of the two most important noise sources in the measurement, which provide the sensitivity limitations in our experiment: Johnson noise and instrumental noise from the input stage of the preamplifier.

Since displacement and force measurements are of utmost importance, we define the spectral noise density for displacement and force detection as $S_{x}$ and $S_{f}$, respectively. What a detector measures is the force. For a resonator mode with quality factor $Q$, the displacement of the resonator near resonance $\omega=\omega_{0}$ is related to the force,

$$
y=\frac{F Q}{k} .
$$


The spectral noise density for force due to the thermal noise can be obtained from the fluctuation-dissipation relation applied to the damped harmonic oscillator systems. At high temperatures, $k_{B} T \gg \hbar \omega_{0}$, the spectral density has the form,

$$
S_{F}^{\text {Johnson }}=\sqrt{\frac{4 k_{B} T k}{\xi Q \omega_{0}}}=\frac{L B}{R_{m}} \sqrt{4 k_{B} T R_{m}} .
$$

The parameter $\sqrt{4 k_{B} T R_{m}}$ is the spectral density for voltage noise with an effective resistance $R_{m}$. The mechanical resistance $R_{m}$ for nanomechanical beams is typically between $0.05 \Omega$ and $10 \Omega$. (We are using the range of values for typical samples, which are micron-long beams with natural frequencies in the range of $\mathrm{MHz}$ with a force constant of 1 $\mathrm{N} / \mathrm{m}$ in a magnetic field of $8 \mathrm{~T}$.) The effective voltage noise density is $<0.2 \mathrm{nV} / \sqrt{\mathrm{Hz}}$ even at room temperature. At temperatures around $10 \mathrm{~K}$, the thermal Johnson noise is much too small to effect the measurement. The dominant noise in the measurement arises due to the voltage noise of the input stage of the first preamplifier, connected to the electrode. The typical value of this noise density $S_{V}^{\text {preamp }}$ is on the order of $1 \mathrm{nV} / \sqrt{\mathrm{Hz}}$. The corresponding force noise is

$$
S_{F}^{\text {instrument }}=\frac{B L}{R_{m}} S_{V}^{\text {preamp }} .
$$

The typical force noise density is $<1 \mathrm{fN} / \sqrt{\mathrm{Hz}}$ at temperatures below $10 \mathrm{~K}$.

\section{Temperature measurement}

The temperature of the resonator is the crystal temperature $T_{\text {crystal }}$, which is determined by the measurement of the electrode temperature $T_{\text {electrode }}$. The gold electrode is evaporated on top of the resonator, making it a resistance thermometer with the closest-possible thermal contact with the crystal. The difference between $T_{\text {crystal }}$ and $T_{\text {electrode }}$ is given by the Kapitza resistance between the two surfaces and the surface area of contact. From the magnetoresistance measurement of the electrode, we have determined the resonator temperature down to $60 \mathrm{mK}$. Above $4 \mathrm{~K}$ the difference between $T_{\text {crystal }}$ and $T_{\text {electrode }}$ due to the Kapitza resistance is $\ll 1 \mathrm{mK}$. We have estimated the temperature difference between the silicon (or GaAs) and gold surfaces with a Kapitza conductance $\sigma_{K}$ of $20 \mathrm{wK}^{-4} \mathrm{~m}^{-2}$ with an area $A$ of $\sim 10 \mu \mathrm{m}^{2}$, and an input power $P$ on the order of $20 \mathrm{fW}$. In this estimate we have used the relation,

$$
T_{\text {crystal }} \simeq\left(\frac{P}{\sigma_{K} A}+T_{\text {electrode }}^{4}\right)^{1 / 4} .
$$

\section{DATA}

Each normal mode is observed by sweeping the drive current through the resonance frequency and recording the induced emf. Quality factors and resonance frequencies are obtained by fitting a circuit model for the device to the resonance curve, which is nearly Lorentzian for low frequency modes [Eq. (4)], as shown in Fig. 5. For the measurements described in this paper, the resonators are operated in the

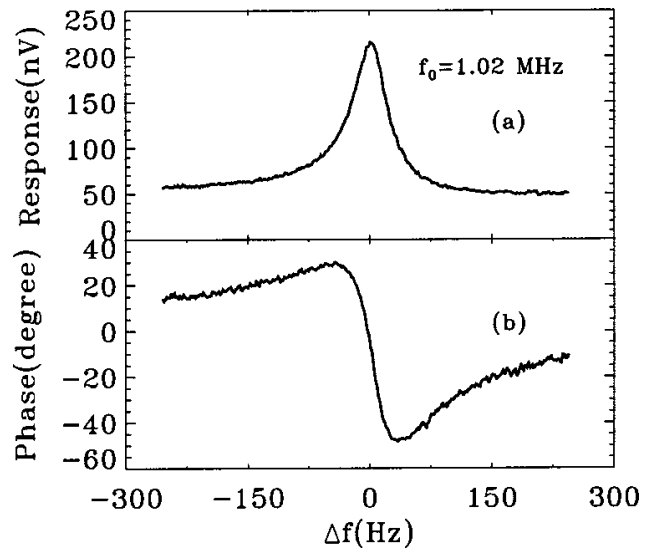

FIG. 5. Measured response to an applied ac drive: (a) the induced emf generated in the gold electrode due to the motion, and (b) the corresponding phase.

linear regime where the motion (or the induced emf) is linearly proportional to the driving field.

In Fig. 6 we display the measured dissipation $Q^{-1}$ in the first three modes between 6 and $90 \mathrm{~K}$ for a typical GaAs resonator with flexural and torsional modes. The trends observed in the temperature dependence are seen in all other samples to be discussed shortly. First, dissipation in the modes shows hysteresis; the temperature dependence traces vary slightly from up-sweeps to down-sweeps even though the overall trends are the same. The maximum difference between two temperature sweeps is found to be on the order of $10 \%$. All other features of the traces are otherwise identical. Second, we observe a peak around $30 \mathrm{~K}$ in the mode A and $\mathrm{B}$. The peak was absent in the lowest dissipation mode C. The high temperature part of the data (above $40 \mathrm{~K}$ ) is described by a power law in temperature with a power of 1 or higher. Because of the small temperature range it is diffi-

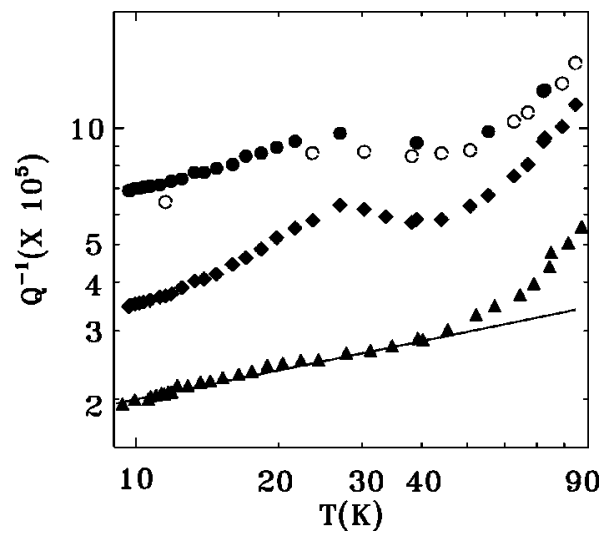

FIG. 6. Typical temperature dependence of dissipation $Q^{-1}$ for three normal modes of a resonator. The open circles represent the dissipation in mode $\mathrm{A}$ and the filled circles represent the same data on a different temperature sweep, suggesting hysteresis. In most of our GaAs samples a thermal activation peak is observed around 30 $\mathrm{K}$, pronounced in the dissipation in mode $\mathrm{B}$ (diamonds). The solid line is a power law fit, $T^{-1 / 4}$, to the data in mode C (triangles). For low dissipation modes, as in mode $\mathrm{C}$, the contribution from the thermal peak around $30 \mathrm{~K}$ is found to be less prominent. 


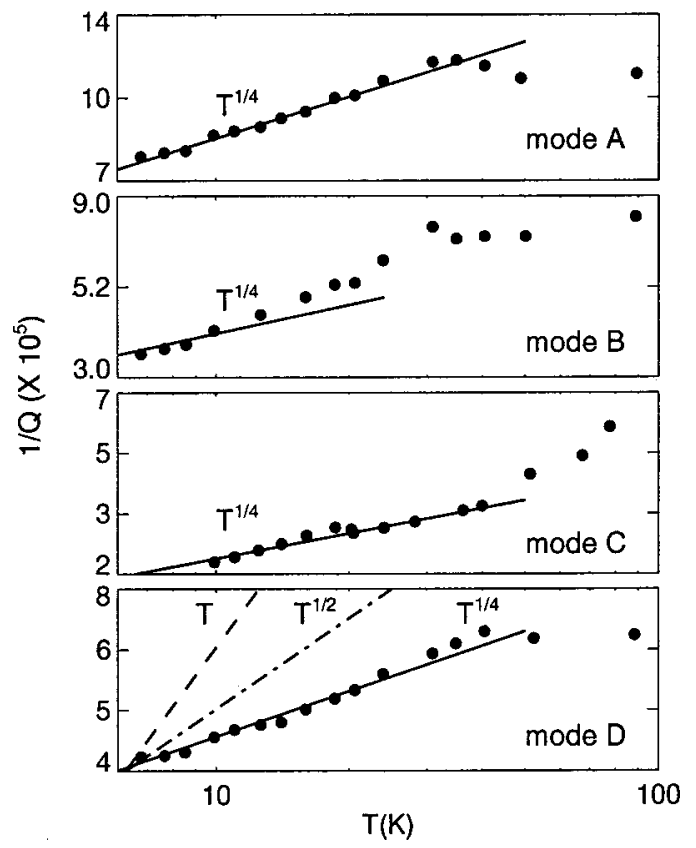

FIG. 7. Temperature dependence of dissipation $Q^{-1}$ for the four normal modes of the resonator. The solid line is a fit to $T^{-1 / 4}$. In the bottom panel, the dashed lines are the power law dependencies, $T^{1 / 2}$ and $T$, shown for comparison.

cult to assign the appropriate power. The low temperature part of the data, however, shows a weakly varying temperature dependence. Fitted to a power law, it is described by $Q^{-1} \propto T^{-1 / 4}$. The solid line in Fig. 7 is the fit to $T^{-1 / 4}$ for the data in mode C. In modes A and B, a weakly temperature dependent background is observed as well.

To better understand the observed trends in this sample, we have studied a set of six GaAs resonators fabricated from the same wafer. Figure 7 displays the temperature dependence of dissipation in the four modes of one of the sample, representative of the set of six. The peak around $30 \mathrm{~K}$ and a weak temperature dependence at lower temperatures are observed in all the samples. The corresponding shift in the resonance frequency $\delta f / f$, is shown in Fig. 8. All the samples show the saturation of resonance frequency below $20 \mathrm{~K}$. The data in Figs. 7 and 8 are taken at a field of $8 \mathrm{~T}$. The temperature dependence measured at different fields are found to be the same, although at a fixed temperature $Q^{-1}$ depends quadratically on magnetic field. The overall trend in temperature appears to be independent of magnetic field (Figs. 9 and 10).

In addition, we also present data for two $\mathrm{Si}$ beams and one GaAs beam with resonance frequencies of $12.4 \mathrm{MHz}, 4.7$ $\mathrm{MHz}$, and $13 \mathrm{MHz}$ for comparison. The temperature dependence shown in Fig. 11 shows the same trend observed in our galium arsenide samples. Below $10 \mathrm{~K}$ the dissipation $Q^{-1}$ saturates in all the three samples. The $\Delta f / f$ data for these samples, shown in Fig. 11, display the saturation of resonance frequency below $20 \mathrm{~K}$, consistent with the data for galium arsenide samples.

We find that both $Q^{-1}$ and $\delta f / f$ are hysteretic on thermal cycling. We have measured the temperature dependence of $Q^{-1}$ and $\delta f / f$ in up-sweeps (by increasing the temperature,

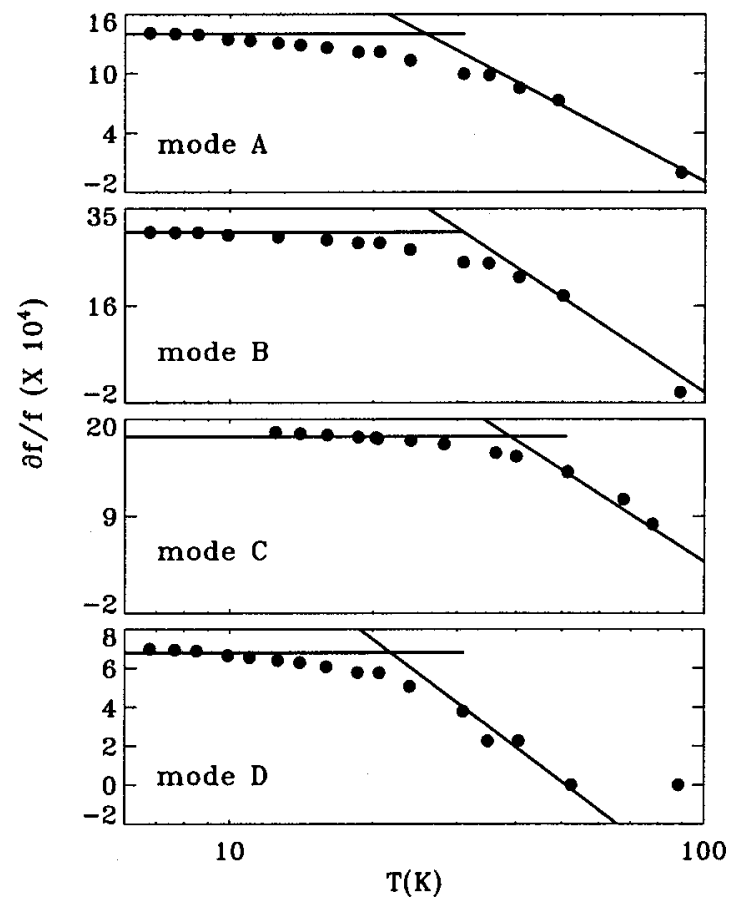

FIG. 8. Temperature dependence of the shift in resonance frequency in a semi-log scale. The high temperature part is fitted to a $\ln T$-form. The low temperature part of the data clearly shows the saturation, starting at a temperature as high as $30 \mathrm{~K}$.

starting at the lowest temperature), and down-sweeps. The values of $Q^{-1}$ and $\delta f / f$ obtained by an up-sweep and a down-sweep during initial thermal cycling are different. Figure 12 displays the difference in the dissipation and shift between an up-sweep and a down-sweep for the $12.4 \mathrm{MHz}$ silicon beam. A peak is observed around $40 \mathrm{~K}$ in the difference of dissipation; there is a small peak at $40 \mathrm{~K}$ in the

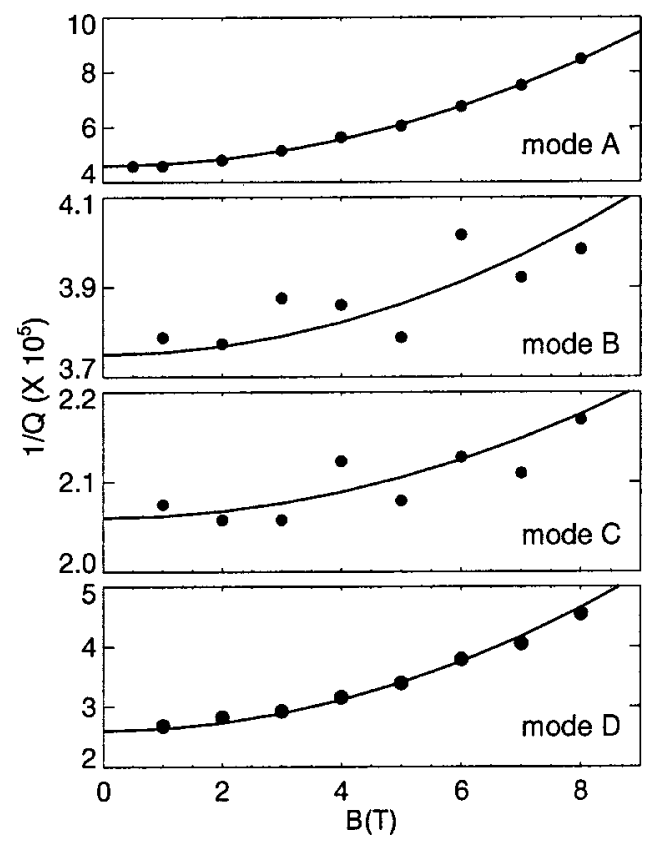

FIG. 9. Magnetic field dependence of $Q^{-1}$ in the four modes. The solid line is a fit to $B^{2}$ form. 


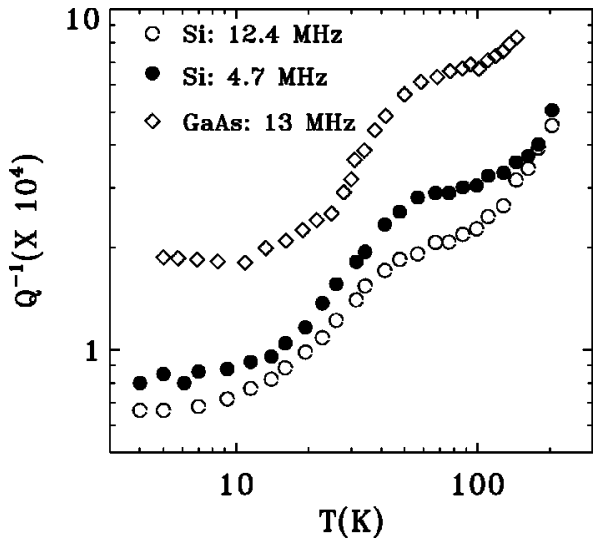

FIG. 10. Temperature dependence of dissipation $Q^{-1}$ for three doubly-clamped beams.

dissipation data as well. The maximum change is observed to be on the order of $10 \%$. It is important to note that the observed hysteresis is not due to a possible lag between the resonator temperature and the thermometer reading.

\section{EXTRINSIC MECHANISMS}

It is essential to verify that the observed dissipation in our mechanical systems is not due to various artifacts as discussed below in detail.

\section{A. Clamping loss}

Loss due to clamping occurs because of the strain at the connection to the support structure. Our measured dissipation is not dominated by clamping loss for the following reasons: (a) $Q^{-1}$ is clearly temperature and magnetic field dependent in every mode of every device measured, whereas clamping loss is expected to be both temperature and magnetic field independent; (b) Mode $\mathrm{C}$ or the decoupled mode of the two-element torsion resonator, shown in Fig. 4, is expected to be less prone to clamping loss since the strain in this mode is essentially localized in the torsion rods connecting the inner and outer elements. Except for the activation peaks, the temperature dependence of $Q^{-1}$ and $\Delta f / f$ for mode $\mathrm{C}$ and other modes are similar, which indicates that

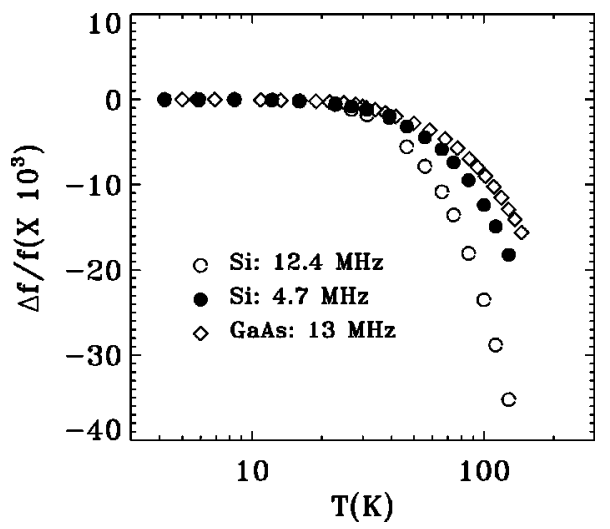

FIG. 11. Temperature dependence of $\Delta f / f$ for the three doublyclamped beams shown in Fig. 10.
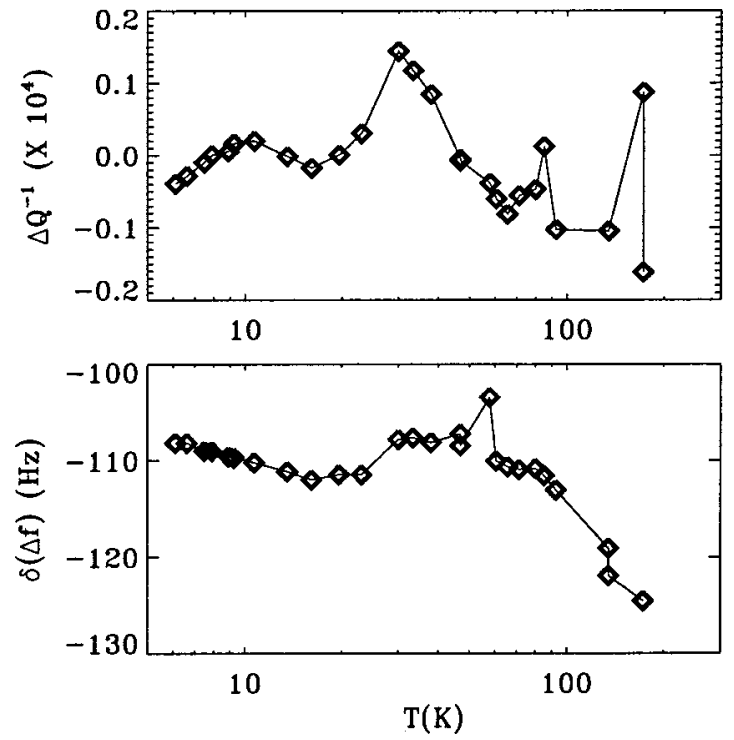

FIG. 12. Hysteresis observed in the dissipation in a silicon beam with a resonance frequency of $12.4 \mathrm{MHz}$. (a) The difference in the dissipation between an up-sweep and a down-sweep shows distinct peaks, coinciding in temperature with the thermal activation peak. (b) The difference in the frequency shift between the two sweeps is displayed.

dissipation in all the modes is not dominated by clamping loss; (c) An experimental study of the size dependence of dissipation, in mode $\mathrm{C}$, reveals that dissipation $Q^{-1}$ increases linearly with the decreasing size of the inner rod, becoming independent of size once the rod width reaches a characteristic value of a micron. This would indicate the importance of the large surface-to-volume ratio in these submicron-sized resonators and the finite size effect becoming dominant. Such a dependence argues against the dominance of clamping loss.

\section{B. Heating}

Temperature was measured by a standard carbon-glass resistance thermometer, and confirmed by the resistance change in the gold electrode on the surface of the device, serving as a secondary thermometer. However, this is not necessarily the device temperature because of finite-though extremely small - Kapitza resistance between $\mathrm{GaAs}$ and $\mathrm{Au}$ surfaces. However, the estimated temperature rise for a typical input power of a picowatt at the surface of the samples is $<1 \mathrm{mK}$, negligible in our temperature range. We can safely neglect heating due to magnetomotive drive and mechanical dissipation, because all measurements are taken in the linear regime where the shape of the resonance curve is independent of the drive amplitude. There was no loss of thermal contact between the sample and the cryostat in the range of 6-100 K because of discernibly strong temperature dependence of $Q^{-1}, \delta f / f$, and the resistance of the Au electrode.

\section{Nonlinearity}

In our experiment, both $Q$ and $\Delta f / f$ are obtained in the linear regime, where the response is independent of the applied drive amplitude. 

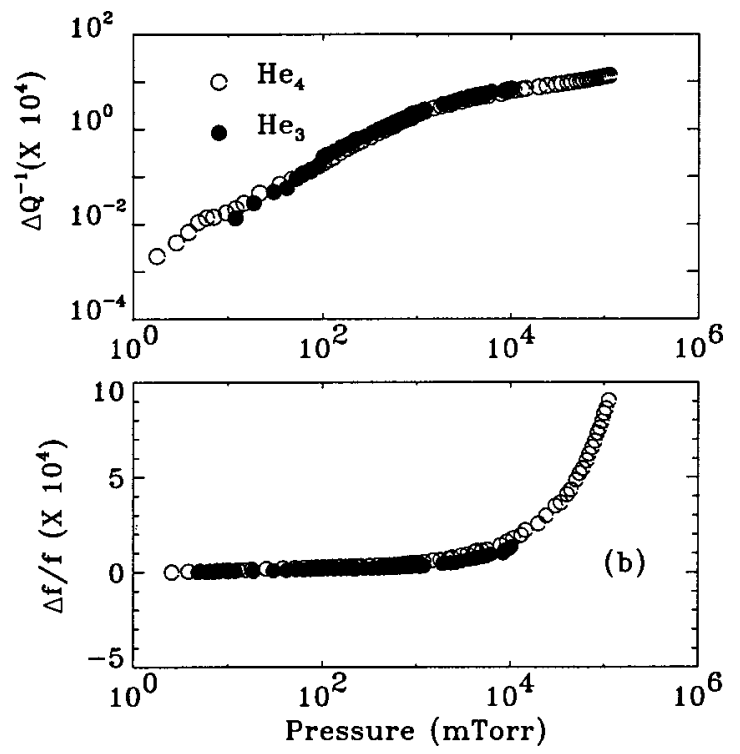

FIG. 13. Pressure dependence of dissipation $Q^{-1}$. There is no detectable change in $Q$ up to an applied pressure of 100 mTorr of either $\mathrm{He}_{3}$ or $\mathrm{He}_{4}$. Note that the pressure in our vacuum can is on the order of $10^{-2}$ mTorr at room temperature, and four orders of magnitude smaller at $10 \mathrm{~K}$.

\section{Gas friction}

We have done detailed measurements and calculations of the dependence of $Q^{-1}$ and $\Delta f / f$ on the gas pressure inside the chamber. A representative data set from gas friction measurements on GaAs beams with $1 \mathrm{MHz}<f<5 \mathrm{MHz}$, which agree with similar measurements done on macroscopic resonators, is displayed in Fig. 13. The quality factor depends linearly on the pressure at the ideal gas limit. For higher pressures, the dissipation is dominated by the excitation of sound waves in the gaseous environment and depends less strongly on the pressure. The first regime and the crossover into the second regime are apparent in our data. Similarly, the resonance frequency is not affected in the ideal gas regime and shifts due to the mass loading of the viscous environment in the hydrodynamic regime.

It is evident from the data that the dissipation increase due to the gas, $\Delta Q^{-1}$, is insignificant for nano- and micromechanical resonators with $Q<10^{5}$ for pressures as high as 100 mTorr for most gases. A simple calculation shows that at a given pressure, the dissipation due to gas friction becomes smaller and smaller as the resonance frequency becomes larger. ${ }^{37}$ Therefore, high frequency resonators are less susceptible to gas friction than their low frequency counterparts.

\section{E. Anharmonic mode coupling}

Coupling between various resonator modes allows the loss of energy from a given mode into others, either irreversibly or on timescales short compared to the Poincare recurrence time. Anharmonicity is crucial to mode coupling, however, our measurements are all done in a linear regime with no detectable mode-coupling. This suggests that the observed dissipation is not due to mode coupling.

\section{F. Thermoelastic loss}

A propagating acoustic wave causes expansion and contraction in an elastic solid, creating a local temperature field. The coupling of the strain field to this temperature field provides a relaxation mechanism where energy is lost from the acoustic mode irreversibly. (a) Though this thermoelastic relaxation is very important for flexural modes at high temperatures, below $100 \mathrm{~K}$, the dissipation rate for micron-sized $\mathrm{GaAs}$ and $\mathrm{Si}$ resonators is found ${ }^{16}$ to be more than three orders of magnitude less than our observed dissipation of $Q^{-1} \sim 10^{-5}-10^{-4}$. (b) Thermoelastic loss is expected to be absent for torsional modes B and C. (c) The observed nonmonotonic temperature dependence, shown in Figs. 7 and 10 cannot be explained by this mechanism which predicts a linear dependence on $\mathrm{T}$, assuming a constant linear thermal expansion coefficient. (d) Thermoelastic loss is independent of magnetic field, whereas our observed dissipation in flexural modes is strongly magnetic field dependent as shown in Fig. 9. For all these reasons, we believe that thermoelastic loss is not relevant in explaining our data.

\section{G. Surface roughness}

There are two basic mechanisms of dissipation which occur due to surface roughness. (a) First, interaction between the acoustic mode with the surface waves, or the Raleigh waves, causes temperature dependent energy loss. However, the observed temperature dependence of $Q^{-1}$ is much slower in our entire temperature range than the expected form of $T^{4}$. (b) Rearrangement of the surface over a long time scale can also give rise to dissipation. We did not observe any structural rearrangements on time scales of days. At a fixed temperature, the resonance frequency of the resonator in any given mode is found to be unchanged, suggesting the lack of significance of any such rearrangement. Magnetic field dependence and various other trends argue against these mechanisms as well.

\section{H. Extrinsic noise}

We can also neglect the influence of the gold electrode in our analysis, for two reasons. First, mode $\mathrm{C}$, in which strain is localized in a metal-free region, shows the same temperature dependence of dissipation as the modes for which the gold electrode is highly strained. Second, the relative resistance change in the Au electrode is measured with an accuracy of $3 \times 10^{-4}$, and its temperature dependence is the same as expected from electron-phonon scattering in this temperature range. The temperature dependence of the resistance is not compatible with $Q^{-1}(T)$ and $\delta f / f(T)$. In addition, GaAs samples with intrinsically doped electrode of $n+$ GaAs layer, with no external gold electrodes, also show similar behavior. This argues against any significant role played by the gold electrodes.

\section{Dislocations}

Internal friction due to intrinsic dislocations ${ }^{38}$ is unlikely to be important in our structures. The usual features of the dislocation-induced internal friction are absent in our data. 
For example, our measurements are linear, and $Q^{-1}$ is independent of the drive amplitude. The shifting of the $Q^{-1}(T)$ trace (anticipated from the depinning of the dislocation) is not observed when the resonator is driven into the nonlinear regime. $Q^{-1}$ is magnetic field dependent. In addition, it is unlikely that all the samples studied would have the same density of dislocations in the relevant submicron regime (the inner rod) where strain is localized. At still higher amplitude, no breakaway (of expected kinked dislocations) is observed.

\section{J. Other material-dependent phenomena}

The temperature dependence of the material properties such as thermal expansion also cannot explain the temperature dependence of $\delta f / f$ and $Q^{-1}$. Our estimate for GaAs shows that the temperature dependence of these material properties results in a contribution too small to explain the observed behavior.

It is clear that the observed trends in the temperature and magnetic field dependence of the dissipation $Q^{-1}$ and the shift in the resonance frequency $\Delta f / f$ are not due to any of the artifacts considered in this section. In the following section we argue that the internal defects in the single crystal resonators contribute dominantly to the observed dissipation.

\section{INTERNAL DEFECTS, $D-x$ CENTERS AND TWO-LEVEL SYSTEMS}

We believe that the observed dissipation in our samples is dominated by the interaction of the resonant acoustic mode with localized defects. Metastable defects on the surface or in the bulk can arise from a variety of reasons: (a) contamination, resulting mainly in water, hydrocarbon and oxide molecules; (b) intrinsic impurity atoms in the bond structure, such as silicon atom in galium arsenide crystals; and (c) dangling or broken bonds on the surface due to the obvious termination of the crystal structure on the surface. There are two contributions to the change in the energy in the energy landscape (energy vs position of the metastable atom): elastic energy and electronic energy.

If the second type of impurities are electronic in naturemeaning, they contribute to a shift of the donor (or acceptor) levels in the Fermi-energy diagram, then they are known as $D-x$ centers. The existence of bistable substitutional defects even in very high quality GaAs, commonly called $D-x$ centers, has been known for decades. ${ }^{39}$ The internal defects dominating dissipation in our samples are likely to be foreign atoms substituting gallium or arsenic atoms in the crystal structure.

For the following reasons we believe that the internal defects dominate the observed dissipation. (a) General trends in our data can be explained by a dissipation mechanism due to internal defects. For example, the thermal activation peak around $30 \mathrm{~K}$ would indicate defect motion between two metastable states separated by a thermal barrier with energy of $30 \mathrm{~K}$. (b) The quadratic dependence on the magnetic field would indicate the presence of charged defects or electronic donor impurities. Depending on the population of these defects, the field dependence can be stronger or weaker. (c) An estimation of defect concentration in our samples is consistent with the known impurity concentration of the starting materials. (d) Various electronic impurities with the energy scales of $10 \mathrm{~K}-70 \mathrm{~K}$ are known to exist in abundance in GaAs and Si systems. (e) The resonance frequency and dissipation are hysteretic, suggesting the existence of multiple equilibrium configurations for these metastable defects. (f) The linear dependence of dissipation on the size is suggestive of the fact that most of the defects reside on the surface for smaller systems with large surface-to-volume ratio. This would explain the observed linear size dependence of the quality factor.

Donor-related defect centers have been known to exist in semiconductors such as galium arsenide and other III-V compounds. There is a host of point defects in such structures, which occur either due to local lattice distortion and vacancies or due to substitutional impurities. Such defects are responsible for bistable behavior in heterostructures. With respect to electronic transport, the presence of these defects reduce conductivity and induce various instabilities which are observable in electronic conductivity or photoconductivity measurements. ${ }^{40}$

A simple local distortion of the lattice potential corresponds to a complex modification of the microscopic energy landscape, resulting in an enormously complicated phenomenology, including the modification of electrical, thermal and acoustic conductivity. For the purpose of understanding dissipation of acoustic waves by defects we shall limit ourselves to low energy excitations of the shallow defects and not be concerned with various deep level defects because of energy considerations. The first kind of impurities are the so-called $D-x$ centers, " $D$ " pertaining to a donor atom coupled with an unknown defect " $x$." In GaAs the unknown atom comes from various species such as $\mathrm{Si}, \mathrm{Ge}, \mathrm{Sn}, \mathrm{S}, \mathrm{Te}$, and Se. The local potential energy curves depend crucially on the type of the impurity as suggested by a vast body of work done on the detection of the type of impurities in GaAs. Some of the impurity atoms such as oxygen have electronic charge associated with them. For GaAs crystal there are metastable $D-$ $x$ centers $^{41}$ coming from electronic impurity states near the conduction band giving a donor level. For $\mathrm{Al}_{x} \mathrm{Ga}_{1-x} \mathrm{As}$ for $x<0.22$, there is a shallow delocalized level from a substitutional site at $7 \mathrm{meV} \simeq 70 \mathrm{~K}$. In $\mathrm{SiO}_{2}$ there are acceptor levels at energies higher than $0.1 \mathrm{~K}$ and donor levels at energies $>10 \mathrm{~K}$.

A very important aspect of most low-energy point defects in GaAs is bistability. Bistability gives rise to unusual phenomena such as persistent photoconductivity, confirmed in many experiments. The experimental observations are accurately described by a microscopic model of bistability as a tilted double-well potential for the defects in configuration coordinates. The energetics and various symmetry properties, specifically in GaAs, have been extensively studied.

Acoustic dissipation due to the defects ${ }^{42,43}$ can be described in terms of phonon capture from the acoustic field either by single-phonon or multiphonon processes. Phonon capture allows transition between two configurational minima. Without any loss of generality, we constrain the language to the transition of a particle such as an impurity atom 
from one minimum to another separated by a distance $d$. The Hamiltonian of this two-level system in the energy eigenbasis is given by

$$
H_{0}=\frac{1}{2}\left[\begin{array}{cc}
E & 0 \\
0 & -E
\end{array}\right] \text {. }
$$

The effect of a strain $u$ induced by an elastic wave is to induce a perturbation to the Hamiltonian,

$$
H=H_{0}+H_{1}=\frac{1}{2}\left[\begin{array}{cc}
E & 0 \\
0 & -E
\end{array}\right]+\frac{1}{2}\left[\begin{array}{cc}
D & 2 M \\
2 M & -D
\end{array}\right] u,
$$

where the deformation potential is given by $D \simeq 2 \gamma \delta / E$, and the off-diagonal element $M \simeq-\gamma \Delta_{0} / E$ describes the coupling between the two states. $\gamma$ is the coupling constant which relates the change in the asymmetry energy to the local elastic strain tensor $\delta \Delta=2 \gamma \delta u_{i k}$.

At low temperatures relaxation is dominated by onephonon processes, described by the transition from an initial state of the combined system $\left|\Psi_{+} ; 0\right\rangle$ (TLS in upper state, no phonon in the field) to a final state $\left|\Psi_{-} ; \boldsymbol{k}, \alpha\right\rangle$ (TLS in lower state, a phonon of wave vector $\boldsymbol{k}$ and polarization $\alpha$ ). The relevant matrix element is given by

$$
\left\langle\Psi_{-} ; \boldsymbol{k}, j\left|H_{1}\right| \Psi_{+} ; 0\right\rangle=\sqrt{\frac{k}{2 \rho v_{j}}} \gamma_{j} \frac{\Delta_{0}}{E}
$$

$\rho$ is the mass density of the sample; $v_{j}$ is the sound velocity.

Due to the coupling between TLS and the elastic field, the TLS causes dispersion and absorption of the acoustic wave. The linear response of the TLS to the elastic strain induced by the acoustic field can be described by a susceptibility function $\xi(\omega)$. The sound dispersion and absorption are given by

$$
\begin{gathered}
\delta v(\omega)=(1 / 2 \rho v) \operatorname{Re}(\xi(\omega)) \\
l^{-1}(\omega)=-\left(\omega / \rho v^{3}\right) \operatorname{Im}(\xi(\omega)) .
\end{gathered}
$$

These two quantities are related to the resonance frequency and the quality factor measured in experiments,

$$
f \propto v ; \quad Q^{-1}=l^{-1} v / \omega,
$$

where $l(\omega)$ is the phonon mean free path.

A wide distribution of asymmetry energies for the similar donor impurity atoms is possible even in a single crystal if the surface to volume ratio of the resonator is large. Since most of the defects are on the surface, the wide distribution is due to the disorder arising from surface roughness. The total contribution to the susceptibility $\xi(\omega)$ is obtained by integrating the contribution of a single two-level system $\xi(\omega)$ over the distribution. Dynamics of the TLS are governed by the Bloch equations, and they describe the following two distinct physical scenarios: resonant and relaxation processes.

The resonant interaction between an acoustic phonon and the TLS results in the absorption of the phonon from the acoustic mode with energy $E=\hbar \omega$. If the upper level is occupied, then the TLS resonantly absorbs the phonon from the acoustic mode, which results in the stimulated emission of two phonons. Since, in the $1 \mathrm{MHz}$ range $E \simeq 40 \mu \mathrm{K}$, all the states are equally populated in the range $6-100 \mathrm{~K}$.

(ii) The relaxation process is caused by the modulation of the asymmetry energy $\delta \Delta$. The acoustic wave, or the strain field, disturbs the thermal equilibrium of the ensemble of the TLS by driving it into a nonequilibrium state. This nonequilibrium state then relaxes to a new equilibrium state with a relaxation rate $1 / \tau$, through interaction with the phonon bath, or with electrons. Relaxation absorption attains a maximum when the frequency of the acoustic wave is $\omega=1 / \tau$.

\section{DISSIPATION DUE TO TWO-LEVEL SYSTEMS}

Before discussing the results of a theory of the dissipation due to the TLS and comparing with experimental results, let us briefly outline the two main motivations for such an analysis. First, (a) for both $\mathrm{GaAs}$ and $\mathrm{Si}$, existence of impurity bands in the range of $10-70 \mathrm{~K}$ is known. This is further corroborated in our experimental data by activation peaks in dissipation, $Q^{-1}$, in this temperature range. Second, (b) The use of such a model arrives at an impurity concentration level on the order of $10^{-14} \mathrm{~cm}^{-3}$, significantly less than the impurity concentration of the starting material.

At a given energy the single-phonon relaxation rate can be found by the use of Fermi's Golden Rule. From the matrix element in Eq. (3),

$$
\frac{1}{\tau_{p}}=\left(\frac{\gamma_{l}^{2}}{v_{l}^{5}}+2 \frac{\gamma_{t}^{2}}{v_{t}^{5}}\right) \frac{\Delta_{0}^{2} E}{2 \pi \rho \hbar^{4}} \operatorname{coth}\left(\frac{E}{2 k_{B} T}\right)
$$

Assuming the width of the upper level to be $\tau_{0}^{-1}$, the quality factor is found to be

$$
\begin{aligned}
Q^{-1} & =\frac{\bar{P} \gamma^{2}}{\rho v^{2}} \frac{\omega \tau_{0}}{4 E} \frac{1}{\omega^{2}+\left(k_{B} T / 4 E^{2}\right)^{2} \tau_{0}^{2}}, \quad k_{B} T \gg E ; \\
& =\frac{2}{\rho v^{2}} \frac{\omega \tau_{0}}{\omega^{2}+\tau_{0}^{2}} \frac{1}{k_{B} T} e^{-2 E / k_{B} T}, \quad k_{B} T \ll E .
\end{aligned}
$$

The shift in the resonance frequency is found to be

$$
\begin{gathered}
\frac{\delta f}{f}=\bar{P} \frac{\gamma^{2}}{2 \rho v^{2}} \frac{1}{2 k_{B} T}+\frac{1}{2 k_{B} T} \frac{\left(k_{B} T \tau_{0} / 2 E\right)^{2}}{\omega^{2}+\left(k_{B} T \tau_{0} / 2 E\right)^{2}}, \quad k_{B} T \gg E ; \\
=\frac{\bar{P} \gamma^{2}}{2 \rho v^{2}} \frac{1}{E}, \quad k_{B} T \ll E .
\end{gathered}
$$

The above results are expressed in Table I in a further simplified form. Dissipation $Q^{-1}$ is expected to decrease approximately as $T^{-2}$ at high temperatures with increasing temperature. At low temperature, it is expected to decrease with decreasing temperature as $T^{-1} e^{-2 E / k_{B} T}$. The frequency 
TABLE I. $\delta f / f$ and $Q^{-1}$ from the linear response of a TLS with energy E. $a=n \gamma_{0}^{2} / \rho v^{2}, \quad c=2 a \omega \gamma_{0} /\left(\omega_{0}^{2}+\gamma_{0}^{2}\right)$, and $d=\omega / k_{B}^{2} \gamma_{0}$. The TLS energy is assumed to be much larger than $\hbar \omega$.

\begin{tabular}{lcc}
\hline \hline Property & $E \gg k_{B} T$ & $E \ll k_{B} T$ \\
\hline$\Delta f / f$ & $a / E$ & $a / 2 k_{B} T$ \\
$Q^{-1}$ & $c e^{-2 E / k_{B} T / k_{B} T}$ & $d T^{-2}$ \\
\hline \hline
\end{tabular}

shift however is described by a tanh form, reaching a constant at low temperatures. At high temperatures, $\delta f / f$ goes as $T^{-1}$.

If there is a spread in $\Delta$, then the quality factor is obtained by integrating over the spread $f(\Delta)$ in $\Delta$, assumed here to be a Gaussian,

$$
Q^{-1}=\frac{\gamma^{2}}{\rho v^{2} k_{B} T} \frac{1}{\omega} \int_{0}^{\infty} \frac{\Delta^{2}}{E^{2}} \operatorname{sech}^{2} \frac{E}{2 k_{B} T} \frac{1}{\tau_{p}} f(\Delta) d \Delta .
$$

This roughly generates a linear dependence, $Q^{-1} \propto T$. At higher temperatures, $\omega \tau_{p}<1$, and $Q^{-1}$ decreases slowly with temperature, giving a plateau. The results of a linear response theory for the acoustic dissipation are given in Table I. The frequency shift, $\Delta f / f$ is described by the function $\tanh \left(E / 2 k_{B} T\right)$, which at low temperatures produces a saturation. Similarly, the functional form for $Q^{-1}$ at high temperatures is described by $T^{-2}$, and at low temperatures by $e^{-2 E / k_{B} T} / T$, an almost exponentially decreasing $Q^{-1}$ with decreasing temperature. There is a plateau or a maximum at intermediate temperatures.

The frequency shift data for all our samples can be fit extremely well to the tanh form, which could explain the saturation of frequency shift at low temperatures. In the dissipation data, first of all, a peak in $Q^{-1}$ is observed in the range of 20-50 K, suggesting the dominant energy of the defect. This is in accordance with the known shallow delocalized level from a substitutional site with an energy on the order of $60 \mathrm{~K}$, for aluminum impurities in GaAs, with concentration $x<0.22$ in $\mathrm{Al}_{x} \mathrm{Ga}_{1-x} \mathrm{As}$. $\mathrm{SiO}_{2}$ has acceptor levels at temperatures of $\geqslant 10 \mathrm{~K}$. The temperature at which the dissipation peak is observed is consistent with previous electronic studies. The high temperature data above $60 \mathrm{~K}$ is not explained well in this model, though phonon scattering mechanism may be important in this regime. The explanation of the $B^{2}$ dependence carries over from the last section, implying that some of the TLS are charged. This is consistent with the fact that in GaAs and Si donor impurity atoms are known to exist in our experimental range of temperature. The only glaring aspect of our data, both in GaAs and Si structures, which cannot be explained in this model is the low temperature part of dissipation, notably the apparent saturation of $Q^{-1}$.

\section{DISCUSSION}

\section{A. Finite-size dependence}

Perhaps the most intriguing question in the problem of dissipation is why it increases, approximately, linearly with decreasing size. In recent experiments where the relevant size of the resonator was changed in a controlled way, an additional aspect of the size dependence is noted, that is, the inverse dissipation (quality factor) first increases with increasing size, then it becomes independent beyond a critical size scale. This behavior is pronounced in the experimental observation of Parpia and coworkers in silicon beams, ${ }^{13}$ and Harrington and co-workers ${ }^{19}$ in GaAs resonators.

The observed size dependence could arise, microscopically speaking, from the mechanism of dissipation due to internal defects. Let us consider a structure with $N$ number of defects contributing to dissipation. (We assume that strain is either globally distributed or the number $N$ corresponds to the volume where strain is localized.) If the size of the system is really large, then one can define a density $\rho$ in the statistical sense,

$$
\rho_{V}=\lim _{V \rightarrow \infty, N \rightarrow \infty} \frac{N}{V} .
$$

For small structures, however, this is not true as the thermodynamic limit is not achieved, and the surface contribution becomes important, and, sometimes, even dominant,

$$
\begin{aligned}
& N \simeq \rho_{S} S+\rho_{V} V, \\
& \frac{N}{V}=\rho_{S} \frac{S}{V}+\rho_{V} .
\end{aligned}
$$

Here, $\rho_{S}$ signifies the number of defects per unit area, and $S / V$ denotes the surface to volume area. In the large volume limit, one indeed obtains the bulk distribution. In the opposite limit of large $S / V$ ratio, the number of defects increases linearly with $S / V$. If one assumes that the internal defects or TLS are not correlated, then the total dissipation $\left(Q^{-1}\right)$ is proportional to the concentration. As a function of the $S / V$ ratio,

$$
Q^{-1} \propto \frac{S}{V} \sim \frac{1}{\text { relevant dimension }} .
$$

This would explain the typical trend observed in recent experiments. Physically, $\rho_{S}$ could represent a variety of surface-related defect mechanisms. These could be contaminant atoms or molecules, dangling bonds on the surface, impurity atoms or molecules which are part of the crystal structure of the starting material-all metastable with timedependent motion on a time scale on the order of the inverse frequency of the structure.

As the size of the system increases, the concentration and hence the dissipation approaches the bulk value. As a function of the size, dissipation first increases linearly, and then it saturates beyond a critical size. Such a saturation has been observed in the experiments of Harrington and co-workers. ${ }^{19}$ Note that this explanation is consistent with the dependences on other parameters such as the magnetic field and temperature.

Now we consider a subtle point regarding the experimental data of dissipation as a function of length of a series of straight beams, shown in Fig. 3. A proper study of the size dependence requires the measurement of dissipation as a 
function of the appropriate system size. In case of beams with varying lengths it is difficult to disentangle the intrinsic dissipation from extrinsic mechanisms such as clamping loss and thermoelastic loss. The specific geometry of beam structure makes it difficult to discern these two mechanisms in the absence of measurements of temperature or magnetic field dependence. Thermoelastic loss occurs in flexural motion when there is a volume change during the expansion or contraction of the beam, and the length dependence in this mechanism is found to be linear. ${ }^{16}$ We conjecture that the linear dependence shown in Fig. 3 is perhaps due to thermoelastic loss. However, a proper study should involve torsional motion where there is no thermoelastic loss due to the lack of volume change during motion. Our concentration in this paper is therefore on structures like the one shown in Fig. 4.

\section{B. Surface, surface-aggregated defects, and TLS}

Recent experiments including ours indicate the dominant role of the surface in ultrasmall resonators. Even though in many papers the relevance of surface has been emphasized, no further microscopic understanding of the effect has been done. Here we point out a general misconception that surface roughness gives rise to enhanced dissipation. A static rough surface will not give rise to dissipation, because dissipation or energy loss occurs only due to a time-dependent potential. To be specific, the rate of energy loss in the standard framework of Fermi's Golden Rule is determined by the matrix element of the time-dependent scattering potential. A timedependent potential is formed either by a dangling bond, defects or impurity atoms moving back and forth between two metastable positions either inside the structure or on the surface, and contaminant atoms or molecules on the surface with an appropriate time dependence, among other possibilities. The lowest energy excitation of all of these can be modeled as a two-level system.

Annealing experiments have revealed that contaminations result in a variety of surface-aggregated metastable foreign molecules, including water, hydrocarbon and oxide molecules. At low energies, the motion of loosely-connected molecules can be modeled as a two-level system, if one considers the lowest-energy metastable state, and the next energy-level metastable state. These molecules can be removed by annealing at very high temperatures.

A different kind of internal defects can also be located on the surface. These are the inherent bulk defects, where a foreign atom replaces a host atom in the bond structure, for example, aluminum replacing a silicon atom in a silicon crystal. However, if the structure size is made smaller and surface-to-volume ratio is made larger, then these bulk defects lie on the surface-they can be called surface defects. Should that be the case, then the dependence of dissipation on size will first increase linearly, and then saturate at large sizes where the number of bulk defects inside the structure dominates over the number of bulk defects on the surface. This saturation effect differentiates between the two surfaceaggregated effect; contamination and replacement of host atom by a foreign atom or molecule in the bond structure.
We consider the former as an experimental artifact, and the latter dominates and still gives a size dependence after the high-temperature heat treatment or anneling of the surface.

\section{Temperature dependence}

Both $Q^{-1}$ and $\delta f / f$ in most of the samples displayed a weak temperature dependence at the low temperature part of the data. The high temperature part of the data as well as the thermal activation peaks could be explained on the basis of thermal transition of internal defects among the metastable positions. Dissipation decreases with decreasing temperature as the thermal population of the higher levels of the defects is reduced. The peak at around $30 \mathrm{~K}$ corresponds to a maximum in the distribution of barrier energies. GaAs and $\mathrm{Si}$ samples are known to have slow fluctuators in the relevant temperature range.

The weak temperature dependence can also be understood with the assumption of noninteracting two-level systems. The thermodynamic arguments are not contained within the framework of the single TLS model described up to this point. In the next section we detail our arguments leading to the explanation of the weak temperature dependence or the saturation.

\section{Magnetic field dependence}

The quadratic dependence of $Q^{-1}$ on magnetic field could be understood assuming that some or most of the defects or TLS are charged. The donor impurity atoms naturally carry an electronic charge in GaAs or Si. The motion of a charged atom with charge $q$ is inhibited in presence of a magnetic field $B$, since the effective momentum scattering rate is modified; $1 / \tau_{m} \rightarrow 1 / \tau_{m}-i \omega_{c}$, where $\omega_{c}=q B / m$ is the cyclotron frequency of the charged atom. The scattering cross section increases by a factor $\left(1+\left(\omega_{c} \tau_{m}\right)^{2}\right)$. This effectively gives a quadratic dependence for dissipation,

$$
Q^{-1}(B)=Q^{-1}(B=0)\left(1+\left(q \tau_{m} / m\right)^{2} B^{2}\right) .
$$

From the coefficient of the quadratic term, it is possible to deduce the momentum scattering rate. For a typical impurity atom like aluminum with a single electronic charge, the scattering time is on the order of tens of nanoseconds. A much more tenable explanation of the $B^{2}$ dependence is based on thermodynamic arguments of entropy and symmetry that in the leading order charged TLS or dipoles would yield a quadratic dependence of dissipation. ${ }^{44,45}$

\section{E. Hysteresis and aging}

A typical concentration of impurities in the starting material (GaAs) is on the order of $10^{16} / \mathrm{cm}^{3}$. This translates into typically $10^{2}-10^{3}$ for a typical micron-sized resonator considered in this paper. Each impurity or defect atom may have many metastable positions. On different cooldowns, the impurity atom relazes to any of these metastable states. Thus under thermal cycling a large number of macroscopic states for the ensemble of impurity atoms is possible, corresponding to the various microscopic states for the individual atoms. If the number of these atoms is extremely small, such 
as the case in our samples, then statistical averaging over the macrostates is not entirely possible. The fluctuation around the mean state can be large and detectable as hysteresis (see Fig. 12). The fluctuation between a typical up sweep and down sweep is on the order of $10^{-4}$. For a typical number of $10^{4}$ impurity atoms, this corresponds to a fluctuation on the order of one atom. These fluctuations grow with increasing temperature as shown in Fig. 12.

Some of the two-level atoms have metastable states with extremely long lifetimes on the order of days, which makes the approach to the equilibrium configuration rather slow. Experimentally, such a slow approach manifests as aging. Hints of aging with thermal cycling and time have been observed in our experiments. Furthermore, this aging process leading to a near-equilibrium condition rather than an equilibrium one for the ensemble of TLS may also give rise to the saturation of dissipation as described in the next section.

\section{NOTA BENE}

In spite of all the qualitative agreement, the apparent saturation of $Q^{-1}$ and $\delta f / f$ is difficult to understand. Here we make a heuristic argument to explain the lack of temperature dependence. First, the ensemble of defects or the pseudospin states have a large degeneracy as it is possible for the pseudo-spins to rearrange in many different configurations with the same total energy. This is true even for the lowest energy state or the ground state of the ensemble. The configurational entropy arising due to the large number of microscopic states hence becomes extremely important in the loss of heat (or energy). The relaxation of the ensemble or the TLS bath to a huge number of macroscopic states provides a dissipation larger than what would be expected from the single nondegenerate microscopic states for the ensemble.

Dissipation at a certain frequency $\omega$ depends on the rate of relaxation of the TLS bath from a nonequilibrium state to an equilibrium state $\tau^{-1}$. The maximum in most cases is obtained when $\omega=\tau^{-1}$. For higher frequencies, only the larger rates contribute to the dissipation substantially. If the relaxation is mostly thermal, as is the case in our experiment, then the rate $\tau^{-1}$ will be an increasing function of temperature, such as a power law. This implies that the increase of frequency would correspond to the observation of this saturation due to the large configurational entropy at a higher temperature. This might also explain why such a dependence was not seen earlier in kilohertz resonators.

Similar saturation of $\delta f / f$, which is related to the shift in the sound velocity, has also been observed in both dielectric constant and sound velocity ${ }^{46-49}$ of glasses, typically below $100 \mathrm{mK}$. Acoustic dissipation and dielectric relaxation in these systems are understood by the tunneling model of $\mathrm{TLS},{ }^{50}$ as tunneling becomes important at temperatures below $1 \mathrm{~K}$. Phenomenologically, the observation of saturation suggests a low-energy cutoff in the tunnel parameters arising perhaps from an additional mechanism outside the framework of the tunneling model. ${ }^{51}$ The interacting tunneling models do not predict a saturation or a slow temperature dependence in dissipation in glasses. ${ }^{52-54}$ It is not clear that a long range interaction, for example the dipole-dipole-type, will reproduce the saturation of dissipation in single crystals. Other theories such as phonon-assisted tunneling and incoherent tunneling ${ }^{55}$ obtain a temperature dependence from the density of states. Obtaining a saturation of dissipation does not seem possible in these models. Our observation of the saturation of $\delta f / f$ in single crystals at much higher temperatures does not necessarily imply that it is due to the same physics governing the saturation in glasses. However, it is possible that the $\delta f / f$ saturation in glasses is related to the weakly temperature dependent dissipation observed in our systems.

Since both quantities, $\delta f / f$ and $Q^{-1}$, show a weak temperature dependence or saturation, it is possible that the temperature of the system may not be the cryostat temperature. Our earlier arguments suggest that loss of thermal contact in the temperature range of $20 \mathrm{~K}$ is highly implausible. The difference is estimated to be at most $1 \mathrm{mK}$. Most likely, the temperature of the relevant heat bath for the TLS is higher than the cryostat temperature even though the sample is in excellent thermal contact with the cryostat. The two-level systems relax by losing their energy to either electrons or phonons. The relaxation is governed by the coupling as well as the density of electrons and phonons.

It is possible that the two-level systems do not really equilibrate. There are two primary reasons for that. First, the density of electrons or phonons can be substantially small in our samples. Second, the coupling of the two-level systems to these baths in this particular energy range may be weak. The fact that the two-level systems do not reach equilibrium in our experiments even over a long time scale is supported by the observation of hysteresis and slight hints of aging. It is now known that if the system size is large compared to the bath, the relaxation times can be much longer than the experimental timescales. In this case, near-equilibrium or nonequilibrium fluctuations of the baths become highly relevant. Assuming that an effective temperature can be defined in analogy to the equilibrium case, the observed temperature dependence will be described by the effective temperature, which is typically higher than the true equilibrium temperature. Both $\delta f / f$ and $Q^{-1}$, defined by the real and imaginary parts of the susceptibility function, will then show a weak temperature dependence though the scales for the both quantities will be different. Plotted against the effective temperature, these quantities may still be described by one of the models considered above.

What determines the loss of equilibrium? At low temperatures, the relaxation time can be much longer than the experimental time scales. As it has been pointed out in many recent publications, ${ }^{56}$ large systems are seen to be dominated by the nonvanishing fluctuations even in long time scales. This makes the violation of the fluctuation-dissipation relation imminent, though one might argue that the fluctuationdissipation relation and the associated law of detailed balance are truly equilibrium phenomena and they should not be valid in a truly nonequilibrium scenario. The effective temperature arises from the analogy with the equilibrium situation. ${ }^{56}$ We believe that a direct measurement of the temperature from the mechanical Johnson noise of these systems 
will be extremely useful in reinforcing these ideas.

Microscopically speaking, our problem perhaps reduces to one where a harmonic oscillator (the resonator mode) is coupled to a bath of two-level spins. This is the inverse of the standard Caldeira-Leggett model, where a two-level spin is coupled to a harmonic oscillator bath. However, in a recent study $^{57}$ it is found that in the inverse Caldeira-Leggett model of a harmonic oscillator coupled to a spin bath one also finds topological dissipation and decoherence at low temperatures due to the topological entropy of the two-level spins. It is possible to have multiple configurations or a large number of degenerate states at low temperatures. This degeneracy leads to the configurational entropy which provides a natural temperature independent mechanism at low temperatures. This so-called orthogonality blocking at low temperatures is a very general feature of the spin bath theories. ${ }^{57}$ One might conjecture that the universal low temperature saturation observed in dipolar glasses may be due to this novel aspect of the theory.

The two-level systems cause dissipation of the acoustic vibration described by harmonic-oscillator modes. It is equivalent to consider the dissipation of the two-level-system dynamics by its coupling to the phonon bath or the acoustic vibrations. However, consideration of quantum-mechanical dynamics of such a coupled, weakly-dissipative two-level system requires that there is tunnel splitting, and the phonons are localized around it. Both these conditions are satisfied at temperatures below $1 \mathrm{~K}$. Further experimental investigations in millikelvin temperatures will be discussed elsewhere. The results of these experiments are consistent with the hightemperature data (above $4 \mathrm{~K}$ ) reported in this paper.

\section{SUMMARY}

In conclusion, we report the temperature and field dependence of dissipation $Q^{-1}$ and shift in the resonance frequency of micron-sized single-crystal mechanical resonators of GaAs and Si. Ours is the first observation of the ubiquitous anomalies of acoustic properties in the single crystal resonators in the Kelvin range. We find that our data is consistent with other observations of similar trends in macroscopic systems at low temperatures. The weak temperature dependence of dissipation universally in many systems in our experiments is perhaps related to the topological entropy anticipated in spin baths.

\section{ACKNOWLEDGMENTS}

We thank Christian Enss, Stefan Kettemann, Ron Lifshitz, and Philip Stamp for many useful discussions. We gratefully acknowledge the experimental help of Jessica Arlett, X.M. Huang, and Jean Casey. This project is financially supported by DARPA.
*Department of Physics, Boston University, 590 Commonwealth Avenue, Boston, Massachusetts 02215. Electronic address: mohanty@physics.bu.edu

${ }^{1}$ V.B. Braginskii, Systems with Small Dissipation (University of Chicago Press, Chicago, 1985).

${ }^{2}$ P. Mohanty, D.A. Harrington, and M.L. Roukes, Physica B 284288, 2143 (2000).

${ }^{3}$ T.D. Stowe, K. Yasumura, T.W. Kenny, D. Botkin, K. Wago, and D. Rugar, Appl. Phys. Lett. 71, 288 (1997).

${ }^{4}$ J.C. Long, H.W. Chan, and J.C. Price, Nucl. Phys. B 539, 23 (1999).

${ }^{5}$ V.M. Mostepanenko and N.N. Trunov, The Casimir Effect and its Applications (Clarendon, Oxford, 1997).

${ }^{6}$ S.K. Lamoreaux, Phys. Rev. Lett. 78, 5 (1997); 81, 5475(E) (1998); Phys. Rev. A 59, R3149 (1999).

${ }^{7}$ U. Mohideen and A. Roy, Phys. Rev. Lett. 81, 4549 (1998); A. Roy and U. Mohideen, ibid. 82, 4380 (1999); G.L. Klimchitskaya et al. Phys. Rev. A 60, 3487 (1999).

${ }^{8}$ S.K. Lamoreaux, Phys. Rev. Lett. 83, 3340 (1999); U. Mohideen et al., ibid. 83, 3341 (1999); A. Lambrecht and S. Reynaud, quant-ph/9912085 (unpublished).

${ }^{9}$ R. Onofrio and G. Carugno, Phys. Lett. A 198, 365 (1995).

${ }^{10}$ M.F. Bocko and R. Onofrio, Rev. Mod. Phys. 68, 755 (1996).

${ }^{11}$ A.N. Cleland, and M.L. Roukes, in Proceedings of the 24th International Conference on the Physics of Semiconductors (World Scientific, Singapore, 1999).

${ }^{12}$ K.Y. Yasumura, T.D. Stowe, E.M. Chow, T. Pfafman, T.W. Kenny, B.C. Stipe, and D. Rugar, J. Microelectromech. Syst. 9, 117 (2000)

${ }^{13}$ S. Evoy S, D.W. Carr, L. Sekaric, A. Olkhovets, J.M. Parpia, and
H.G. Craighead, J. Appl. Phys. 86, 6072 (1999).

${ }^{14}$ D.W. Carr and H.G. Craighead, J. Vac. Sci. Technol. B 15, 2760 (1997).

${ }^{15}$ D.A. Harrington, P. Mohanty, and M.L. Roukes, Physica B 284288, 2145 (2000).

${ }^{16}$ R. Lifshitz and M.L. Roukes, Phys. Rev. B 61, 5600 (2000).

${ }^{17}$ R.E. Mihailovich and J.M. Parpia, Phys. Rev. Lett. 68, 3052 (1992).

${ }^{18}$ D.W. Carr, S. Evoy, L. Sekaric, H.G. Craighead, and J.M. Parpia, Appl. Phys. Lett. 75, 920 (1999).

${ }^{19}$ D.A. Harrington and M.L. Roukes (unpublished).

${ }^{20}$ A.N. Cleland and M.L. Roukes, Appl. Phys. Lett. 69, 2653 (1996).

${ }^{21}$ D.S. Greywall, B. Yurke, P.A. Busch, and S.C. Arney, Europhys. Lett. 34, 37 (1996).

${ }^{22}$ S. Evoy, A. Olkhovets, L. Sekaric, J.M. Parpia, H. Craighead, and D.W. Carr, Appl. Phys. Lett. 77, 2397 (2000).

${ }^{23}$ A. Olkhovets, S. Evoy, D.W. Carr, J.M. Parpia, and H.G. Craighead, J. Vac. Sci. Technol. B 18, 3549 (2000).

${ }^{24}$ J.L. Yang, T. Ono, and M. Esashi, Appl. Phys. Lett. 77, 3860 (2000); J.L. Yang, T. Ono, and M. Esashi, Sens. Actuators A A82, 102 (2000).

${ }^{25}$ M. Cross and R. Lifshitz, cond-mat/0011501 (unpublished).

${ }^{26}$ R.N. Kleiman, G. Agnolet, and D.J. Bishop, Phys. Rev. Lett. 59, 2079 (1987).

${ }^{27}$ W.A. Phillips, Phys. Rev. Lett. 61, 2632 (1989).

${ }^{28}$ R.W. Keyes, Phys. Rev. Lett. 62, 1324 (1989).

${ }^{29}$ T. Klistner and R.O. Pohl, Phys. Rev. B 36, 6551 (1987).

${ }^{30}$ W. Duffy, J. Appl. Phys. 68, 5601 (1987).

${ }^{31} \mathrm{G}$. Agnolet (unpublished). 
${ }^{32}$ R.A. Buser and N.F. de Rooij, Sens. Actuators A 21, 323 (1990).

${ }^{33}$ I. Tittonen, G. Breitenbach, T. Kalkbrenner, T. Muller, R. Conrad, S. Schiller, E. Steinsland, N. Blanc, and N.F. de Rooij, Phys. Rev. A 59, 1038 (1999).

${ }^{34}$ J.J. Yao and N.C. Macdonald, J. Micromech. and Microeng. 5, 257 (1995).

${ }^{35}$ D.F. Mcguigan, C.C. Lam, R.Q. Gram, A.W. Hoffman, D.H. Douglass, and H.W. Gutche, J. Low Temp. Phys. 30, 621 (1978).

${ }^{36}$ A.N. Cleland and M.L. Roukes, Sens. Actuators A 72, 256 (1999).

${ }^{37}$ K.L. Ekinci and M.L. Roukes (to be published).

${ }^{38}$ A. Seeger, J. Phys. (Paris) 42, 201 (1981).

${ }^{39}$ P.M. Mooney, Semiconductors 51, 93 (1999); Physics and Application of Defects in Semiconductors, edited by M.O. Manasreh (Materials Research Society, New York, 1994); Imperfections in III/V Materials, Semiconductors, and Semimetals, edited by E.R. Weber (Academic, New York, 1993), Vol. 38; T.N. Theis, P.M. Mooney, and B.D. Parker, J. Electron. Mater. 20, 35 (1991).

${ }^{40}$ V. Narayanamurti, R.A. Logan, and M.A. Chin, Phys. Rev. Lett. 43, 1536 (1979); D.V. Lang and R.A. Logan, ibid. 39, 635 (1977); D.V. Lang, R.A. Logan, and M. Jaros, Phys. Rev. B 19, 1015 (1979).

${ }^{41}$ D.J. Chadi and K.J. Chang, Phys. Rev. B 39, 10063 (1989).

${ }^{42}$ W. Phillips, J. Low Temp. Phys. 7, 351 (1972); Amorphous Solids: Low-Temperature Properties, edited by W.A. Phillips (Springer-Verlag, Berlin, 1981).

${ }^{43}$ From Coherent Tunneling to Relaxation: Dissipative Quantum Dynamics of Interacting Defects, Alois Wrger, Springer Tracts in Modern Physics (Springer, Berlin, 1997), Vol. 135.

${ }^{44}$ M.J. Naughton, S. Dickinson, R.C. Samaratunga, J.B. Brooks, and K.P. Martin, Rev. Sci. Instrum. 54, 1529 (1983).

${ }^{45}$ S. Kettemann, P. Fulde, and P. Strehlow, Phys. Rev. Lett. 83, 4325 (1999).

${ }^{46}$ D. Salvino, S. Rogge, B. Tigner, and D.D. Osheroff, Phys. Rev. Lett. 73, 268 (1994); S. Rogge, D. Natelson, and D.D. Osheroff, ibid. 76, 3136 (1996); S. Rogge, D. Natelson, B. Tigner, and
D.D. Osheroff, Phys. Rev. B 55, 11256 (1997); D. Natelson, D. Rosenberg, and D.D. Osheroff, Phys. Rev. Lett. 80, 4689 (1998).

${ }^{47}$ J. Classen, C. Enss, C. Bechinger, G. Weiss, and S. Hunklinger, Ann. Phys. (Leipzig) 3, 315 (1994); J. Classen, M. Hubner, C. Enss, G. Weiss, and S. Hunklinger, Phys. Rev. B 56, 8012 (1997); G. Weiss, M. Hubner, J. Classen, C. Enss, and S. Hunklinger, Physica B 220, 305 (1996).

${ }^{48}$ C. Enss and S. Hunklinger, Phys. Rev. Lett. 79, 2831 (1997); P. Strehlow et al., ibid. 80, 5361 (1998). Note that this mechanism is inherently different from the crossover mechanism of coherent to incoherent tunneling in the spin-boson model described in A. Leggett, S. Chakravarty, A.T. Dorsey, M.P.A. Fisher, A. Garg, and W. Zwerger, Rev. Mod. Phys. 59, 1 (1987).

${ }^{49}$ E. Thompson, R.O. Pohl, and J. Parpia, Phys. Rev. Lett. 84, 4601 (2000).

${ }^{50}$ P.W. Anderson, C.M. Varma, and B.I. Halperin, Philos. Mag. 25, 1 (1972).

${ }^{51}$ Tunneling Systems in Amorphous and Crystalline Solids, edited by Pablo Esquinazi (Springer, Berlin, 1998).

${ }^{52}$ A. L. Burin, D. Natelson, D.D. Osheroff, and Yu. Kagan, in Ref. 51; See also, A.L. Burin and Yu. Kagan, Sov. Phys. JETP 80, 761 (1995); 109, 299 (1996).

${ }^{53}$ A.J. Leggett, Physica B 169, 322 (1991).

${ }^{54}$ C.C. Yu, Phys. Rev. Lett 63, 1160 (1989); C.C. Yu, Comments Condens. Matter Phys. 14, 231 (1988).

${ }^{55} \mathrm{See}, \mathrm{A}$. Wurger in Ref. 51 for a review.

${ }^{56}$ S. Franz, M. Mezard, G. Parisi, and L. Peliti, Phys. Rev. Lett. 81, 1758 (1998); J-P. Bouchaud, L.F. Cugliandolo, J. Kurchan, and M. Mezard, in Spin-Glasses and Random Fields, edited by A. P. Young (World Scientific, Singapore, 1998); also as cond-mat/9702070; L.F. Cugiandolo, J. Curchan, and L. Peliti, Phys. Rev. E 55, 3898 (1997); L.F. Cugliandolo and G. Lozano, Phys. Rev. B 59, 915 (1999).

${ }^{57}$ N.V. Profok'ev, and P.C.E. Stamp, Rep. Prog. Phys. 63, 669 (2000). 